\title{
Rolling Bearing Fault Diagnosis Based on Component Screening Vector Local Characteristic-Scale Decomposition
}

\author{
Tengfei Guan (D), Shijun Liu, Wenbo Xu, Zhisheng Li, Hongtao Huang, and Qi Wang \\ Zhengzhou Research Institute of Mechanical Engineering Co., Ltd, Zhengzhou 45000, China \\ Correspondence should be addressed to Tengfei Guan; guantengfei1314@126.com
}

Received 9 April 2021; Revised 1 June 2021; Accepted 23 December 2021; Published 10 January 2022

Academic Editor: Ke Feng

Copyright ( 2022 Tengfei Guan et al. This is an open access article distributed under the Creative Commons Attribution License, which permits unrestricted use, distribution, and reproduction in any medium, provided the original work is properly cited.

\begin{abstract}
The fault vibration signal of a bearing has nonstationary and nonlinear characteristics and can be regarded as the combination of multiple amplitude- and frequency-modulation components. The envelope of a single component contains the fault characteristics of a bearing. Local characteristic-scale decomposition (LCD) can decompose the vibration signal into a series of multiple intrinsic scale components. Some components can clearly reflect the running state of a bearing, and fault diagnosis is conducted according to the envelope spectrum. However, the conventional LCD takes a single-channel signal as the research object, which cannot fully reflect the characteristic information of the rotor, and the analysis results based on different channel signals of the same section will be inconsistent. To solve this problem, based on full vector spectrum technology, the homologous dual-channel information is fused. A vector LCD method based on cross-correlation coefficient component selection is given, and a simulation analysis is completed. The effectiveness of the proposed method is verified by simulated signals and experimental signals of a bearing, which provides a method for bearing feature extraction and fault diagnosis.
\end{abstract}

\section{Introduction}

Rotating machinery is developing in the direction of high speeds, heavy loads, and high reliability, which places higher requirements on mechanical transmission equipment [1]. The operational state of mechanical equipment is changing, and its safe, stable, and reliable operation must be ensured. Rolling bearings are widely used in mechanical equipment, and their working condition greatly affects its operation [2]. Owing to complex operating conditions and changing external environment, rolling bearings are prone to failure [3]. It is of great significance to monitor their working status and diagnose their fault degree $[4,5]$. Some studies have focused on the fault features of rotating machinery through modern signal processing methods [6-8].

Fault vibration signals of bearings are usually weak nonstationary signals with complex frequency components. The key to fault diagnosis of rolling bearings is to extract effective feature information from vibration signals containing complex frequencies [9]. Vibration analysis and fault diagnosis have received considerable attention [10-14] and have been adopted to process nonstationary and nonlinear vibration signals. Among them, signal decomposition methods contribute much. Tiwari [15] described a selfadaptive signal decomposition technique, concealed component decomposition (CCD), as the basis of a precise bearing fault diagnosis model. Ying [16] introduced a novel permutation entropy-based improved uniform phase empirical mode decomposition (PEUPEMD) method and obtained better analysis than comparative methods about empirical mode decomposition (EMD) in decomposing accuracy and mode mixing suppression. Patel [17] applied variational mode decomposition (VMD) to filter out nonstationarities due to variable speed conditions and provided a complete diagnostic solution for the spur gear systems. Li [18] presented a local mean decomposition (LMD) method based on an improved compound interpolation envelope, whose effect was comparable to or slightly better than that of other methods. Zheng [19] proposed local characteristicscale decomposition (LCD), a nonstationary signal analysis method that adaptively decomposes a signal to a series of intrinsic scale components in different scales. With good 
compatibility, LCD methods have seen new applications, such as the local characteristic-scale decomposition-Teager energy operator (LCD-TEO) [20], improved local characteristic-scale decomposition (ILCD) [21], and piecewise cubic Hermite interpolating polynomial-local characteristicscale decomposition (PCHIP-LCD) [22].

However, the conventional LCD methods focus on singlechannel signals, which probably cause incomplete fault feature extraction. In the fault diagnosis of rotating machinery, the sensor information collected by a multi-sensor system is related to the same or different sides of rotating machinery in the same environment. There is an inevitable connection between various types of information, which are different in time, space, credibility, and expression, whose focuses and uses are not exactly the same, and whose requirements for information processing and management are different. If the information collected by each source is considered in isolation, then their internal connections and characteristics are lost. Multi-source information fusion can solve this problem [23]. Multi-sensor data fusion methodologies include the holospectrum [24-27], full spectrum [28-31], and full vector spectrum [32]. Proposed by Han [32], the full vector spectrum has been widely studied and applied in engineering [33-35] and has formed the basis of many compound methods. Chen [36] applied full-vector signal acquisition and information fusion to fault prediction. Gong [37] combined the full vector spectrum with ensemble empirical decomposition and applied it to the diagnosis of gear faults. Yu [38] introduced the empirical wavelet transform and variance contribution rate to the full vector spectrum, which improved the adaptability and accuracy of full vector information fusion.

Based on the above analysis, the main contributions of this paper are as follows:

(1) A signal processing method, vector LCD, is proposed, which fully considers homologous signals and intrinsic scale components (ISCs)

(2) Vector LCD can simplify the analysis of ISCs by taking the cross-correlation coefficient in screening components

(3) The fusion of optimal components can obtain more complete and accurate fault features

The remainder of this article is arranged as follows. Section 2 shows the calculation of LCD, presents the theory of the cross-correlation coefficient, describes the principles of the conventional full vector spectrum, and introduces a method for bearing feature extraction and fault diagnosis based on the correlation coefficient vector LCD. In Section 3, the proposed methodology is verified through application to the homologous simulation signals of a rolling bearing. In Section 4, the rolling bearing experimental data from two directions of sensors are used to validate vector LCD. Conclusions are given in Section 5.

\section{Theoretical Description of Vector LCD}

The proposed fault diagnosis method using vector local characteristic-scale decomposition (Vector LCD) is presented in Figure 1. Vibration signals were obtained from a test stand by a signal acquisition module. Then, the vector LCD method was used to compute and analyze the data. Next, the fusion data were enveloped into the spectrum. Lastly, the fault frequency features were matched with the specific fault type and the failure reason was located.

2.1. Local Characteristic-Scale Decomposition. According to the extreme value of a signal, the LCD can adaptively decompose nonlinear and nonstationary signals to a series of ISCs satisfying the following conditions:

(1) The length between any two adjacent extreme points of the data sample is monotonic

(2) If the extreme point in a data sample is $X_{k}(k=1,2$, ..., M) and $\tau_{k}$ is the corresponding time, then any two maximum (or minimum) value points $\left(\tau_{k}, X_{k}\right)$, $\left(\tau_{k+2}, X_{k+2}\right)$ can be connected to form a line segment. $\tau_{k+1}$ is the corresponding time of the maximum (or minimum) value point $\left(\tau_{k+1}, X_{k+1}\right)$ in the middle of the line segment. The corresponding function value at this moment is

$$
A_{k}=X_{k}+\frac{\tau_{k+1}-\tau_{k}}{\tau_{k+2}-\tau_{k}}\left(X_{k+2}-X_{k}\right) .
$$

The ratio of the function value to the maximum (or minimum)

$$
a\left[X_{k}+\frac{\tau_{k+1}-\tau_{k}}{\tau_{k+2}-\tau_{k}}\left(X_{k+2}-X_{k}\right)\right]+(1-a) X_{k+1}=0,
$$

remains unchanged, where $a \in(0,1)$ is a constant, and $a=1 / 2$ for frequency modulated, amplitude modulated, amplitude-frequency modulated, and sine-cosine signals.

On the basis of the ISC, LCD can decompose any signal $x(t)$ to a series of ISCs, as follows [39]:

(1) Find all extreme points of $x(t)$ and their corresponding moments $\tau_{k}(k=1,2, \ldots, \mathrm{M})$, set $a=1 / 2$, and make a linear transformation for $x(t)$ between any two extreme points,

$$
\begin{aligned}
P_{1} & =L_{k}+\frac{L_{k+1}-L_{k}}{X_{k+1}-X_{k}}\left(x_{t}-X_{k}\right), \\
L_{k+1} & =a\left[X_{k}+\frac{\tau_{k+1}-\tau_{k}}{\tau_{k+2}-\tau_{k}}\left(X_{k+2}-X_{k}\right)\right]+(1-a) X_{k+1},
\end{aligned}
$$

where $t \in\left(\tau_{k}, \tau_{k+1}\right)$.

(2) Subtract $P_{1}(t)$ from the original signal $x(t)$ to get a new signal,

$$
I_{1}(t)=x(t)-P_{1}(t) .
$$

(3) Ideally, $I_{1}(t)$ can be used as the first ISC. At this moment $L_{k+1}$ is equal to zero; in practice, assuming a variable $\Delta e$, the iteration ends when $\left|L_{k+1}\right| \leq \Delta e$. If 


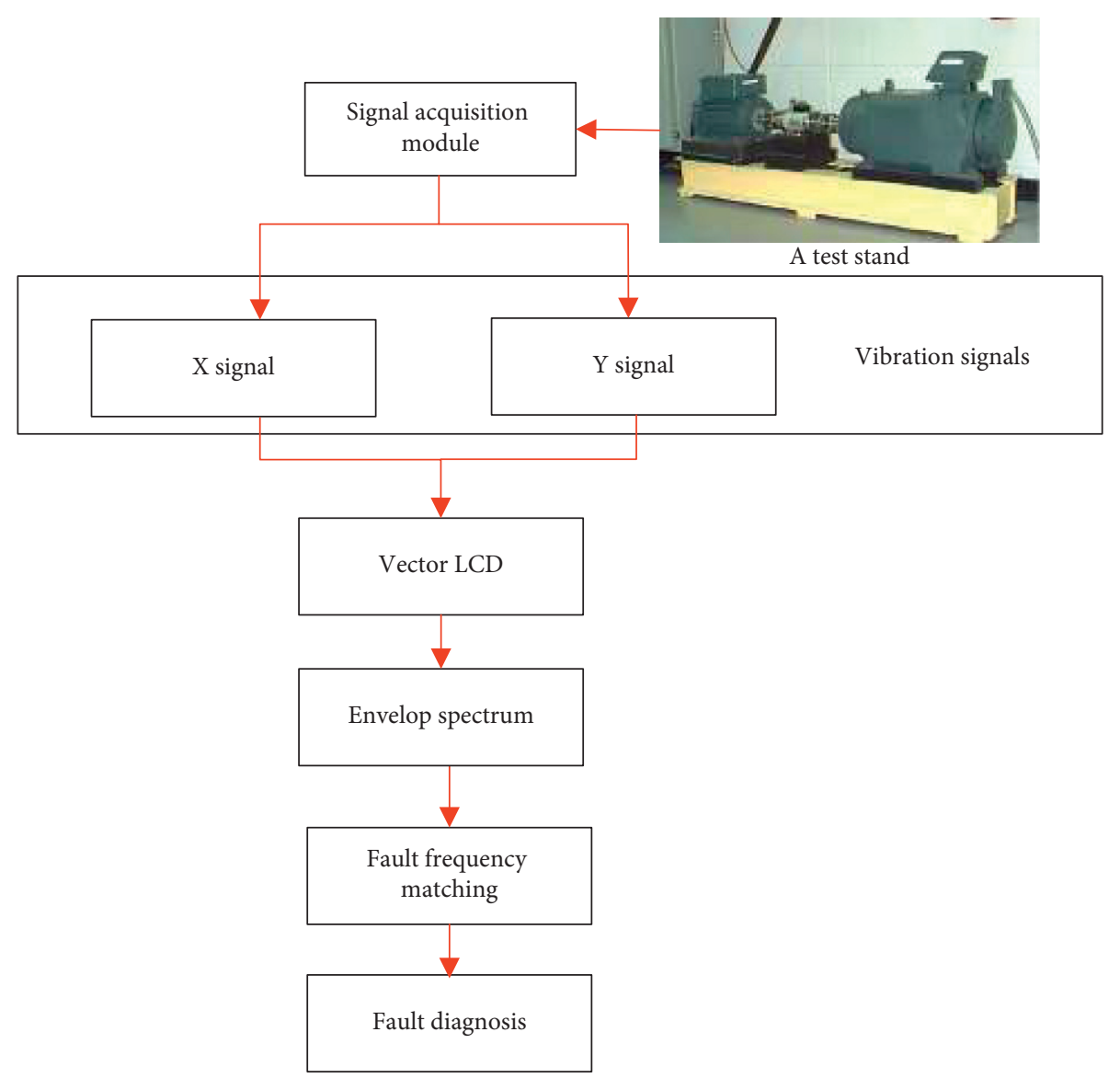

FIGURE 1: Block diagram of the proposed fault diagnosis method using vector LCD.

$I_{1}(t)$ does not meet the two conditions of ISC, the above steps are repeated $k$ times until $I_{k}(t)$ satisfies the conditions and denote $I_{k}(t)$ as the first ISC $c_{1}(t)$ of $x(t)$.

(4) Subtract $c_{1}(t)$ from $x(t)$ to get a new signal, $r_{1}$. Take $r_{1}$ as the original data and repeat steps $1-3$ to get the second ISC component, $c_{2}(t)$, of $x(t)$. Repeat $n$ times to get $n$ ISCs of the signal $x(t)$. The function does not terminate until $r_{n}$ is monotonic:

$$
x(t)=\sum_{p=1}^{n} c_{p}(t)+r_{n}(t)
$$

It can be seen from equation (5) that the signal $x(t)$ can be reconstructed by $n$ ISCs and a monotonic signal.

2.2. Correlation Coefficient. Correlation is a kind of nondeterministic relationship, and the correlation coefficient measures the degree of linear correlation between variables. The correlation coefficient between sequences $x=\left(x_{1}, x_{2}, \ldots, x_{n}\right)$ and $y=\left(y_{1}, y_{2}, \ldots, y_{n}\right)$ can be calculated as

$$
r=\frac{\sum_{i=1}^{n}\left(x_{i}-\bar{x}\right)\left(y_{i}-\bar{y}\right)}{\sqrt{\sum_{i=1}^{n}\left(x_{i}-\bar{x}\right)^{2}} \sum_{i=1}^{n}\left(y_{i}-\bar{y}\right)^{2}},
$$

where $\bar{x}$ and $\bar{y}$ are the mean values of sequences $x$-and $y$, respectively.

Suppose $x_{\text {nor }}$ and $y_{\text {nor }}$ are normal operating signals perpendicular to each other, and $x_{\text {abn }}, y_{\text {abn }}$ are the homologous signals when faults occur. After the decomposition of LCD, we obtain four ISCs, ISC $_{\text {xnork }}$, ISC $_{\text {ynork }}, I_{\text {ISC }}$ yabnk, and ISC $_{\text {xabnk }}$, where $k=1,2, \ldots, N$ is the order of an ISC. The cross-correlation coefficient is calculated as follows:

(1) Calculate the correlation coefficients between $\mathrm{ISC}_{\text {xnork }}$ and $x_{\text {nor }}, \mathrm{ISC}_{\text {ynork }}$, and $y_{\text {nor }}$, and find their average, $U_{k}$;

(2) Calculate the correlation coefficients between ISC $_{\text {xabnk }}$ and $x_{\text {abn }}$, ISC $_{\text {yabnk }}$, and ISC $_{\text {yabnk }}$, and find their average, $V_{k}$;

(3) Calculate the correlation coefficients between ISC $_{\text {xnork }}$ and ISC $_{\text {ynork }}$, ISC $_{\text {xabnk }}$, and ISC $_{\text {yabnk }}$, and find their average, $W_{k}$;

(4) Calculate the sensitivity factor, 


$$
S_{k}=\frac{U_{k}+V_{k}}{2}-W_{k}
$$

where $U_{k}$ and $V_{k}$ indicate the degree of correlation between the decomposed ISC and the initial signal. The larger the value, the more similar are ISC and the initial signal. The $W_{k}$ indicates the correlation between ISCs of the same order. The smaller the value, the greater the change in the ISC. Overall, the larger the $S_{k}$, the more sensitive the ISC of this order, and the more it can reflect spectral changes.

2.3. Full Vector Spectrum. To overcome limitations due to incomplete and inaccurate sensor information, two orthogonal sensors are usually fixed on the same section of the rotor in the field test of large rotating machinery. The full vector spectrum technology meets the accuracy and reliability requirements of condition monitoring and fault diagnosis. The vortex phenomenon of the rotor is the combined effect of each harmonic frequency, and the vortex intensity at each harmonic frequency is the basis for fault judgment and identification. The space rotation trajectory of each harmonic is an ellipse, and the maximum vibration vector is in its long-axis direction [40].

Suppose the cross section channel signals ( $\left\{x_{k}\right\}$ and $\left.\left\{y_{k}\right\}\right)$ are perpendicular to each other and form them into a complex signal $\left(\left\{z_{k}\right\}=\left\{x_{k}\right\}+j\left\{y_{k}\right\}\right)$, only a single Fourier transform (FT) of the complex signal is needed to obtain the characteristic information required by the full vector spectrum under each harmonic frequency. The algorithm is robust, it greatly reduces calculation, and it is compatible with conventional analysis methods. When processing a single-channel signal, the algorithm is still valid and can meet real-time requirements. The characteristic information includes the main vibration vector $R_{L k}$, assistant vibration vector $R_{s k}$, angle $\varnothing_{\alpha k}$ between the main vibration vector and the $x$-axis, and the elliptical trajectory's initial phase angle $\alpha_{k}$, which are described by

$$
\left\{\begin{array}{l}
R_{L k}=\frac{1}{2 N}\left[\left|Z_{k}\right|+\left|Z_{N-k}\right|\right], \\
R_{s k}=\frac{1}{2 N}\left[\left|Z_{k}\right|-\left|Z_{N-k}\right|\right] \\
\tan 2 \varnothing_{\alpha k}=\frac{Z_{I k} Z_{R(N-k)}-Z_{R k} Z_{I(N-k)}}{Z_{I k} Z_{I(N-k)}+Z_{R k} Z_{R(N-k)}} \\
\tan \alpha_{k}=\frac{Z_{I k}+Z_{I(N-k)}}{Z_{R k}+Z_{R(N-k)}}
\end{array}\right.
$$

where $k=1,2, \ldots, \mathrm{N} / 2-1$. The characteristic information of each harmonic trace from equation (8) is the main characteristic information under each harmonic trace of full vector spectrum technology.

Figure 2 is a flowchart of the proposed method. At first, the LCD is applied to the homology information acquired from two orthogonal sensors. The cross-correlation coefficient is selected to choose the ISCs of orthogonal signals, the next optimal ISC of orthogonal signals can be obtained, the vector ISCs are formed through the optimal ISC component fusion, and the vector ISCs are enveloped and demodulated. The proposed method can identify the nonlinear characteristics of fault signals for fault diagnosis.

\section{Simulation Analysis}

Analog signals were analyzed to validate the effectiveness of vector local characteristic-scale decomposition in processing homologous signals. For the rolling bearing signal, the vibration signal at the time of failure presents a modulation phenomenon. The vibration signal of a rolling bearing with an outer ring fixed structure is

$$
x(t)=\alpha \sin \left(2 \pi f_{b}\right)\left(1+\beta \sin \left(2 \pi f_{r} t\right)\right),
$$

where $f_{b}$ is the passing frequency of the inner ring of the rolling bearing and $f_{r}$ is the rotation frequency of the rotor.

Based on this, the following analog acceleration signal is constructed:

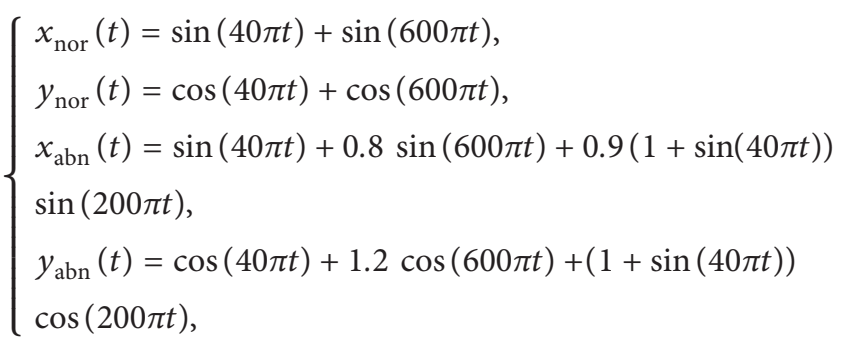

where $x_{\text {nor }}(t)$ and $y_{\text {nor }}(t)$ are two initial vibration signals that are perpendicular to each other under normal operating conditions, $x_{\mathrm{abn}}(t)$ and $y_{\mathrm{abn}}(t)$ are two initial vibration signals that are perpendicular to each other under abnormal operating conditions, the sampling frequency $f_{s}=1024 \mathrm{~Hz}$, and there are $N=2048$ sampling points.

The LCD is applied to decompose the two-channel signals corresponding to normal operating conditions. The time-domain waveforms and the ISCs are shown in Figure 3 for $\mathrm{x}$-channel signals and Figure 4 for $\mathrm{y}$-channel signals.

The LCD is then applied to decompose the two-channel signals corresponding to abnormal operating conditions, for which the time-domain waveforms and the ISCs of $\mathrm{x}$ - and $\mathrm{y}$-channel signals are shown in Figures 5 and 6, respectively.

The correlation coefficients between ISCs and initial signals can be calculated, i.e., between ISC $_{\mathrm{xnori}}$ and $\mathrm{x}_{\text {nor, }}$ ISC $_{\text {ynori }}$ and $\mathrm{y}_{\text {nor, }}$ ISC $_{\mathrm{xabni}}$ and $\mathrm{x}_{\mathrm{abn}}$, and $\mathrm{ISC}_{\mathrm{yabni}}$ and $\mathrm{y}_{\mathrm{abn}}$, as shown in Table 1.

Figures 5 and 6 show that the initial signals are decomposed to six ISCs with different frequency bands. There is a great difference in amplitude between the $\mathrm{x}$ - and $\mathrm{y}$-directions. If the signal is analyzed in a certain direction alone, the analysis results will also be quite different. Therefore, information fusion is necessary. The full vector fusion is introduced. Considering the value of the correlation coefficient, the first-order ISC $_{\mathrm{xnor}}$ and third-order ISC $_{\text {ynor }}$ are closer to the initial signals than the others. The second-order ISC $_{\text {xabn }}$ and second-order ISC $_{\text {yabn }}$ have more 


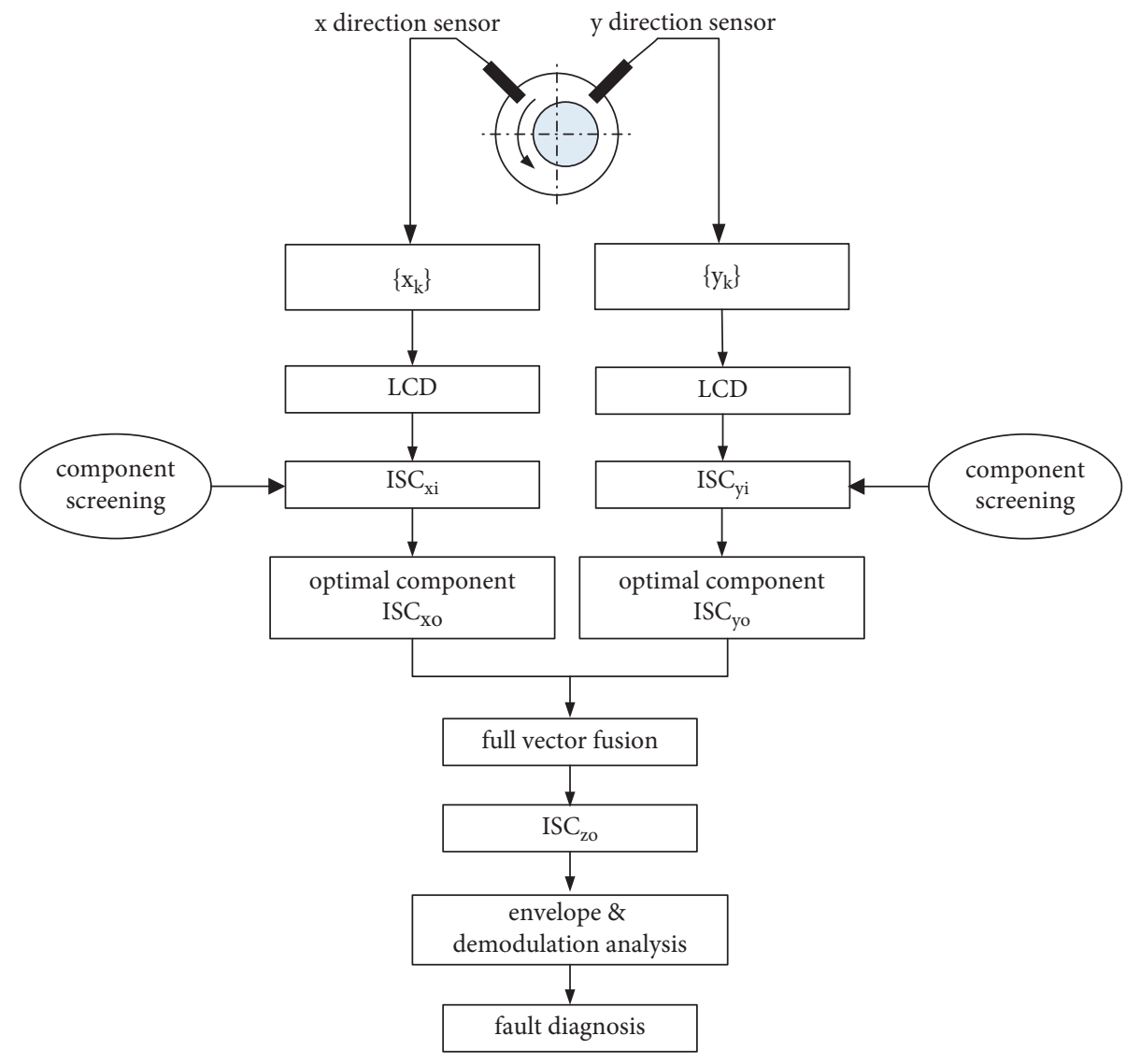

Figure 2: Flowchart of vector LCD.
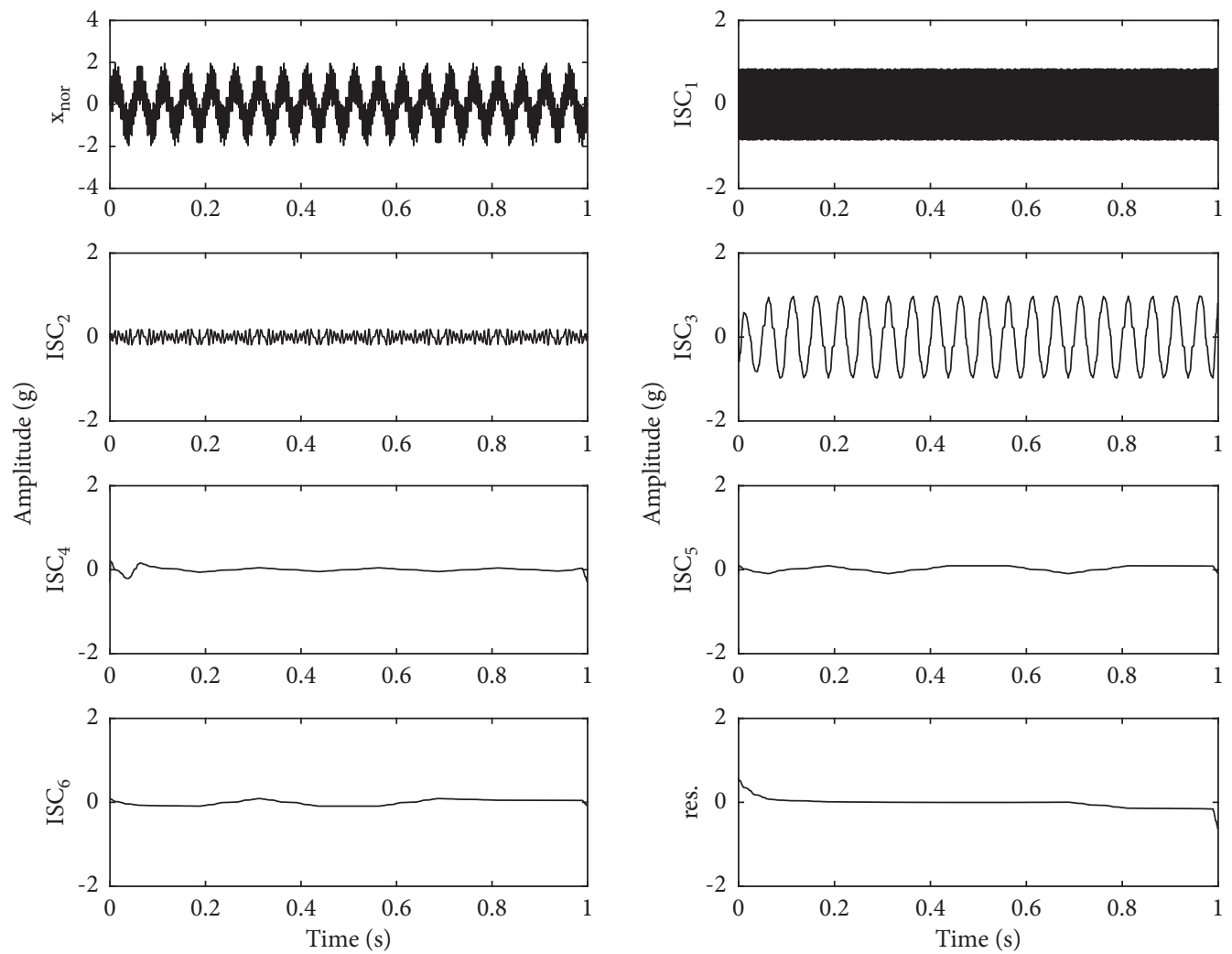

Figure 3: LCD decomposition results of $\mathrm{x}$-channel under normal conditions. 

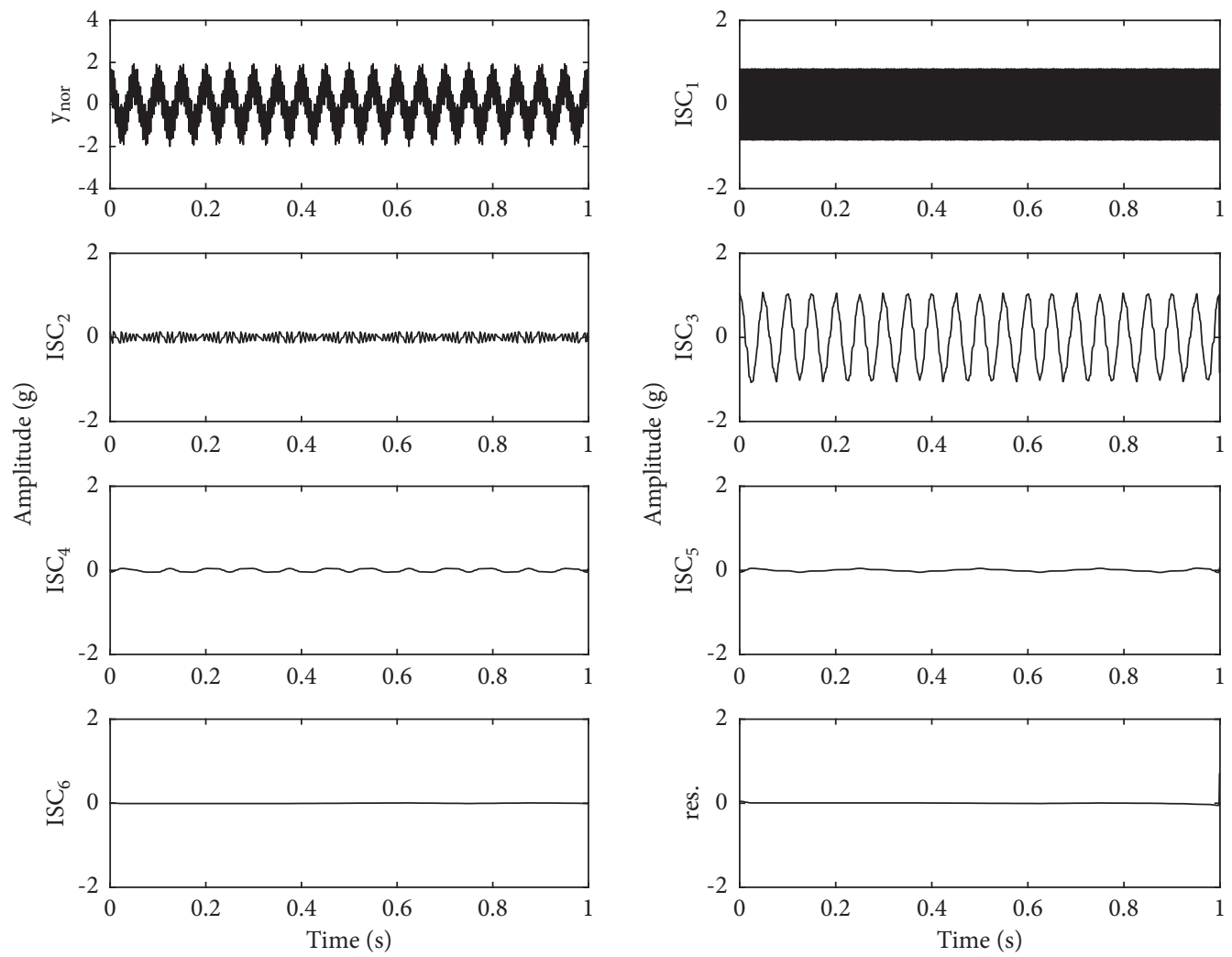

FIGURE 4: LCD decomposition results of y-channel under normal conditions.
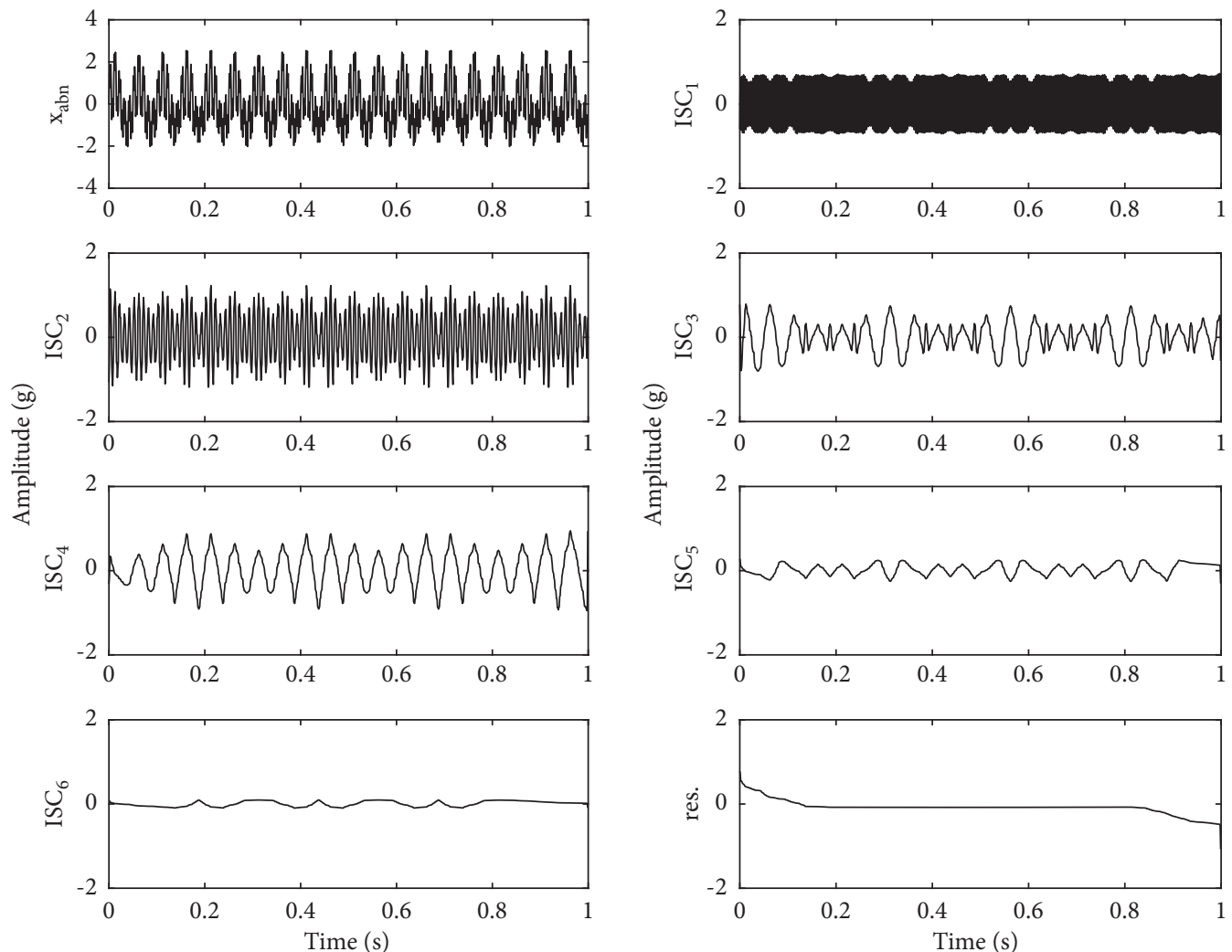

Figure 5: LCD decomposition results of $\mathrm{x}$-channel under abnormal conditions. 

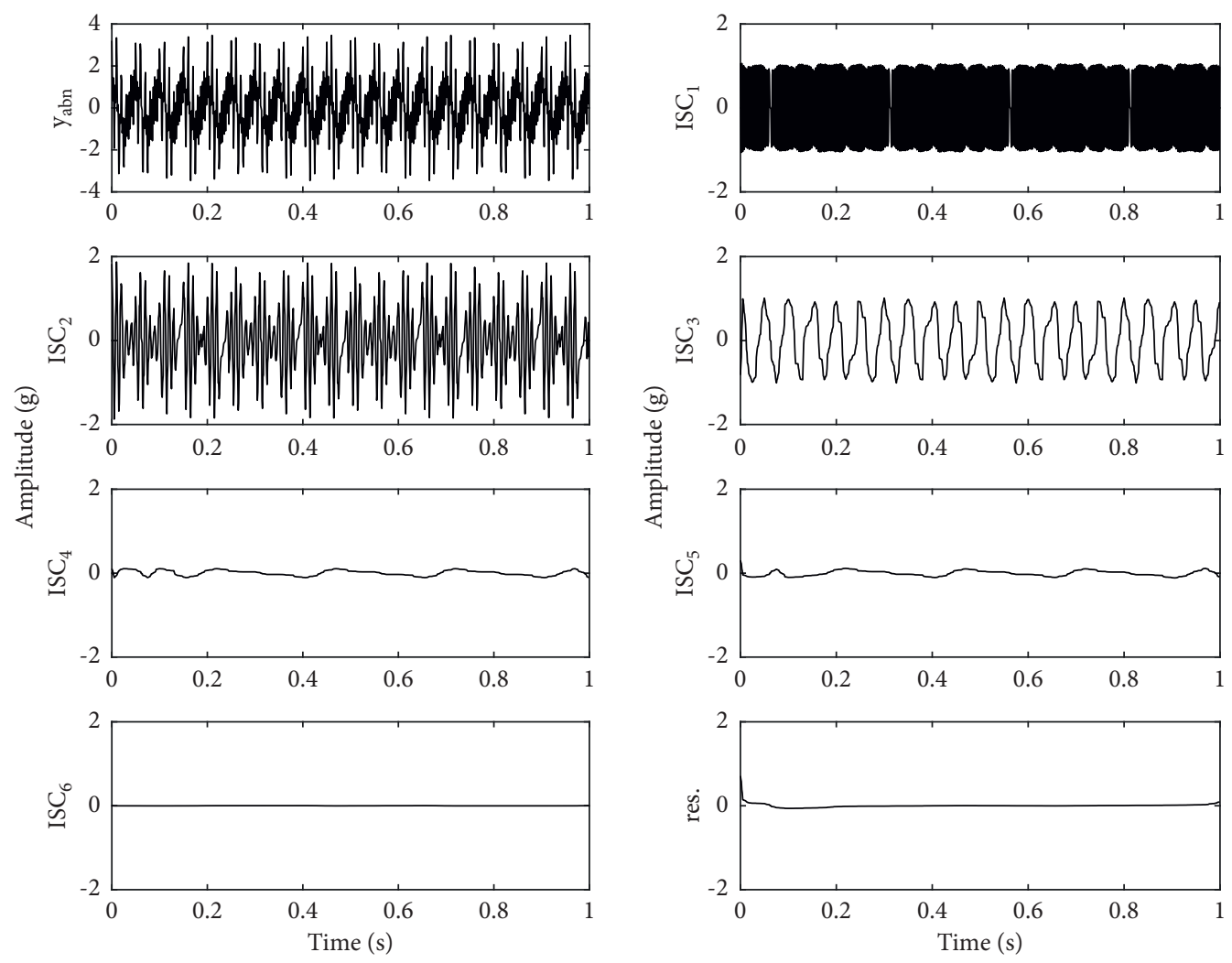

FIGURE 6: LCD decomposition results of y-channel under abnormal conditions.

TABLE 1: Correlation coefficients of ISCs of each order between two channel signals under two conditions.

\begin{tabular}{llcccccc}
\hline ISC order & & 1 & 2 & 3 & 4 & 5 \\
\hline & $\mathrm{x}_{\text {nor }}$ & $\mathbf{0 . 7 0 3 5}$ & 0.1648 & 0.6998 & 0.0869 & 0.0032 & 0.0062 \\
Correlation coefficient & $\mathrm{y}_{\text {nor }}$ & 0.6983 & 0.1851 & $\mathbf{0 . 7 0 4 5}$ & -0.1065 & 0.11 & -0.0203 \\
& $\mathrm{X}_{\text {abn }}$ & 0.5308 & $\mathbf{0 . 6 2 9 3}$ & 0.4658 & 0.6119 & 0.0043 & -0.0628 \\
& $\mathrm{y}_{\text {abn }}$ & 0.6385 & $\mathbf{0 . 6 5 7 6}$ & 0.5345 & 0.0147 & -0.0027 & 0.0125 \\
\hline
\end{tabular}

The bold value is the maximum value of all ISCs (between the order 1 to 6 ).

fault information than others. Thus, we apply full vector fusion to the first-order ISC $_{\text {xnor }}$, third-order ISC ${ }_{\text {ynor, }}$, secondorder $\mathrm{ISC}_{\mathrm{xabn}}$, and second-order ISC $\mathrm{Iabn}_{\mathrm{y}}$. However, the fault frequency information cannot be acquired just from timedomain waveforms. Finally, envelope and demodulation are applied to fusion signals, as shown in Figure 7. It is clear in Figure 7 (a) that the rotation frequencies $(20 \mathrm{~Hz}$ and $300 \mathrm{~Hz})$ are consistent with the preset value under the normal operating condition. In Figure $7(\mathrm{~b})$, the amplitudes of the fault frequency $(100 \mathrm{~Hz})$ and sidebands $(20 \mathrm{~Hz})$ show the existence of modulation under the abnormal operating condition.

From the simulation analysis, the vector LCD has a good analysis result when applied to fault signals with frequency or amplitude modulation, which can enhance the accuracy of fault diagnosis. The adoption of the cross-correlation coefficient avoids repeated analysis between multiple components and simplifies the analysis, for a unique and accurate conclusion.

\section{Application}

The validity and advantage of vector LCD in fault diagnosis of a rolling bearing were examined through experimental data from the Case Western Reserve University bearing data center. Figure 8 shows the layout of the test stand, which consisted of a 2-hp motor, torque transducer/encoder, dynamometer, and control electronics. The test bearings supported the motor shaft, and they had single-point faults from electro-discharge machining. Accelerometers collected vibration data and were attached to the support of the drive end bearing, fan end bearing, and motor supporting base. The technical parameters of fault diagnosis are listed in Table 2.

Normal baseline data, outer race fault data, and inner race fault data were adopted from the experimental signals. The ball fault data frequency is not matched with the usual value for the slip adjusting to lock onto a dominant frequency [41]. Therefore, ball fault data are not discussed here. 


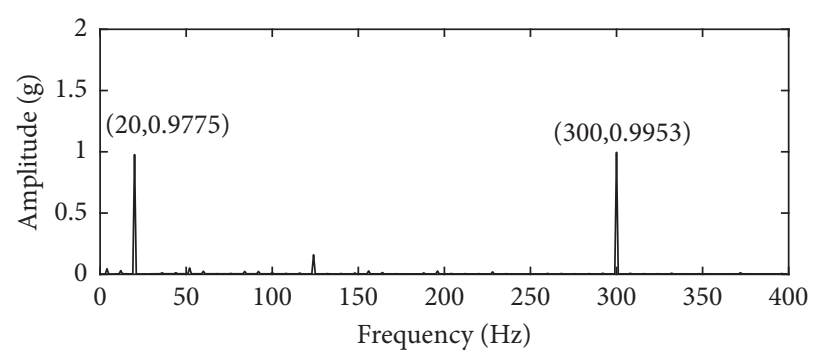

(a)

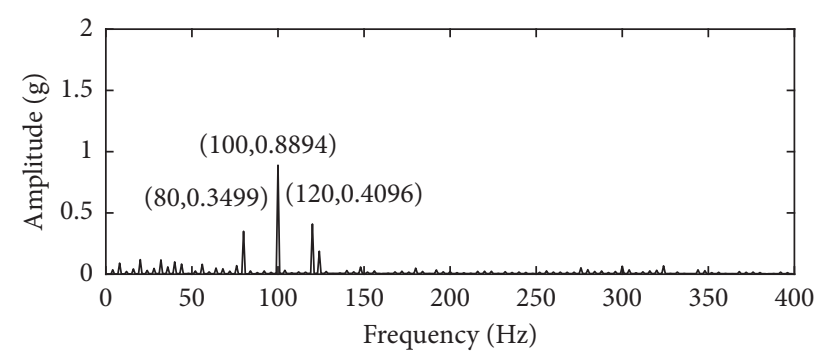

(b)

FIgURE 7: Vector LCD spectra of simulation signals: (a) normal condition; (b) abnormal condition.

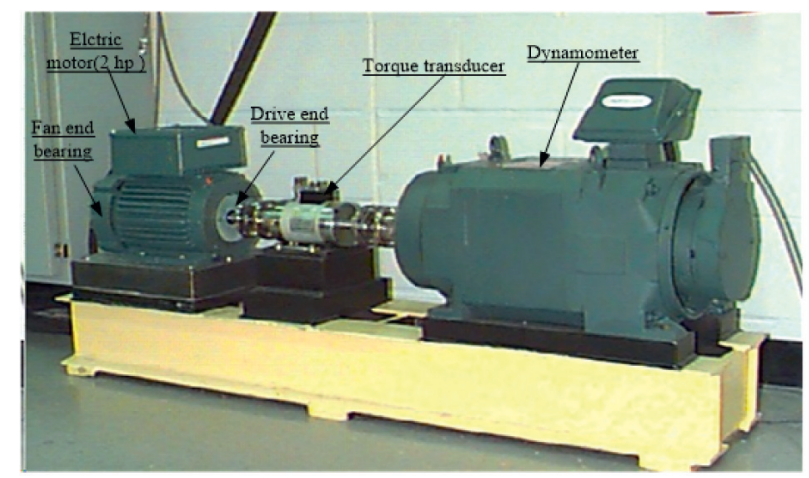

FIGURE 8: Case Western Reserve University bearing test stand.

TABLE 2: Technical parameters.

\begin{tabular}{lcccc}
\hline Fault location & Fault diameter (inches) & Fault depth (inches) & Motor load (hp) & Sampling frequency (Hz) \\
\hline Normal & 0 & 0 & 0 & 12000 \\
Outer race & 0.007 & 0.011 & 0 & 12000 \\
Inner race & 0.007 & 0.011 & 0 & 12000 \\
Fault location & Motor speed (rpm) & Defect frequencies $(\mathrm{Hz})$ & Sample points & Data file name \\
Normal & 1797 & - & 8192 & $97 . \mathrm{mat}$ \\
Outer race & 1797 & 107.3 & 8192 & $130 . \mathrm{mat} / 144 . \mathrm{mat}$ \\
Inner race & 1797 & 162.1 & 8192 & $105 . \mathrm{mat}$ \\
\hline
\end{tabular}

4.1. Normal Baseline Data Analysis. Because the normal baseline data only have one channel of drive end section, processing the single-sensor data is just a special case of a full vector spectrum. According to the flowchart of vector LCD, we first applied LCD to the drive-end single-channel signal to obtain 10 ISCs. Considering that the higher the order of ISC, the smaller the amplitude, only the first six orders of ISCs are displayed in Figure 9. We compare the values of ISC cross-correlation coefficients in Table 3, where the first is the optimal component. Finally, the envelope and demodulation are applied to the first ISC. From Figure 10, the rotation frequency $f_{\mathrm{r}}(29.95 \mathrm{~Hz})$ and $2 f_{\mathrm{r}}(59.9 \mathrm{~Hz})$ account for the main components without other fault frequencies, which is a normal operating condition.

4.2. Outer Race Fault Data Analysis. Outer race fault data were obtained by three homologous sensors in the same section, and channels of centered 6:00 clock and orthogonal 3:00 clocks were employed to vector LCD analysis. Similar to normal baseline data processing, ISC components were acquired after LCD. The ISC components of the $\mathrm{x}$-and $\mathrm{y}$-channels are shown in Figures 11 and 12, respectively. As seen in Table 4, the first ISC components of the $\mathrm{x}$-and $\mathrm{y}$-signals are optimal. From Figures 11 and 12, the time-domain waves of the first ISC components of the $\mathrm{x}$ - and $y$-signals have different amplitudes and frequencies. If only one direction of the $\mathrm{x}$ - or $\mathrm{y}$-signal is analyzed, then different results will be obtained, which is contrary to fault diagnosis. Therefore, we apply full vector fusion to the two optimal ISC components to obtain a new combined signal. Finally, the envelope and demodulation are applied to the combined signal. The outer race fault frequency $\mathrm{f}_{\mathrm{OR}}(107.3 \mathrm{~Hz})$ can be easily distinguished from other spectra, and the amplitude of $\mathrm{f}_{\mathrm{OR}}$ is the highest among all spectra (as seen in Figure 13).

4.3. Inner Race Fault Data Analysis. Inner race fault data were recorded in a single channel for the drive end, and the inner race fault signal was treated in the same way as normal 

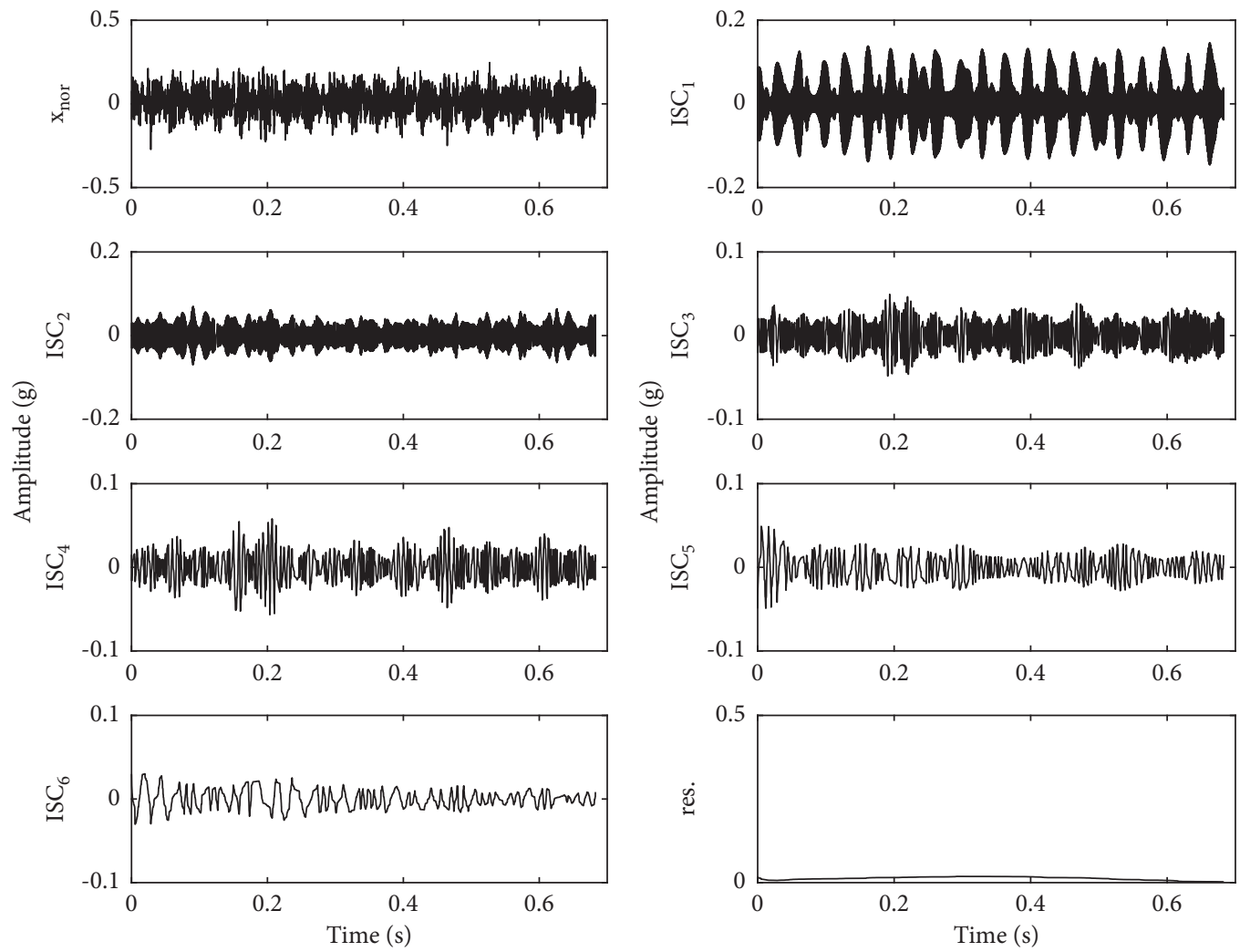

FIGURE 9: LCD decomposition results of $\mathrm{x}$-channel under normal conditions.

TABLE 3: Correlation coefficients of ISCs of each order under normal conditions.

\begin{tabular}{lccccc}
\hline ISC order & 1 & 2 & 3 & 4 & 5 \\
\hline Correlation coefficient & $\mathbf{0 . 7 9 9 4}$ & 0.5678 & 0.3904 & 0.4009 & 9 \\
ISC order & 6 & 7 & 8 & 0.3037 \\
Correlation coefficient & 0.1167 & 0.0773 & 0.0052 & 10 \\
\hline
\end{tabular}

The bold value is the maximum value of all ISCs (between the order 1 to 10).

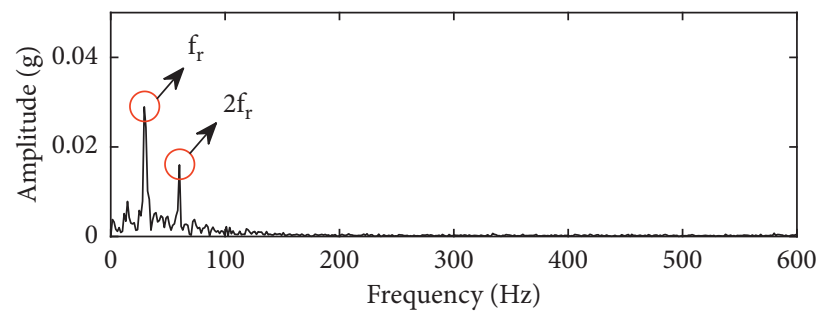

FIGURE 10: Vector LCD spectra of $\mathrm{x}$-channel under normal conditions.

baseline data. The vector LCD spectra can be displayed after the optimal ISC is screened out, with decomposition results as shown in Figure 14. From Table 5, the optimal ISC (the first ISC component) can be easily obtained. From the vector LCD spectra in Figure 15, the inner race fault frequency $\mathrm{f}_{\mathrm{IR}}$ $(162.1 \mathrm{~Hz})$ and rotation frequency harmonics $f_{\mathrm{r}}(29.95 \mathrm{~Hz}), 2 f_{\mathrm{r}}$ $(59.9 \mathrm{~Hz})$, and $4 f_{\mathrm{r}}(119.8 \mathrm{~Hz})$ can be acquired from the spectra, which agrees with the inner race fault features.
This bearing experimental application shows that the proposed method can be applied to rolling bearing fault diagnosis. The screening of the optimal ISC can simplify fault diagnosis and clearly display the typical features. The full vector fusion between optimal ISCs of the $\mathrm{x}$ - and $y$-signals gives an accurate and unique conclusion for fault diagnosis. The vector LCD provides an easy way to extract fault features. 

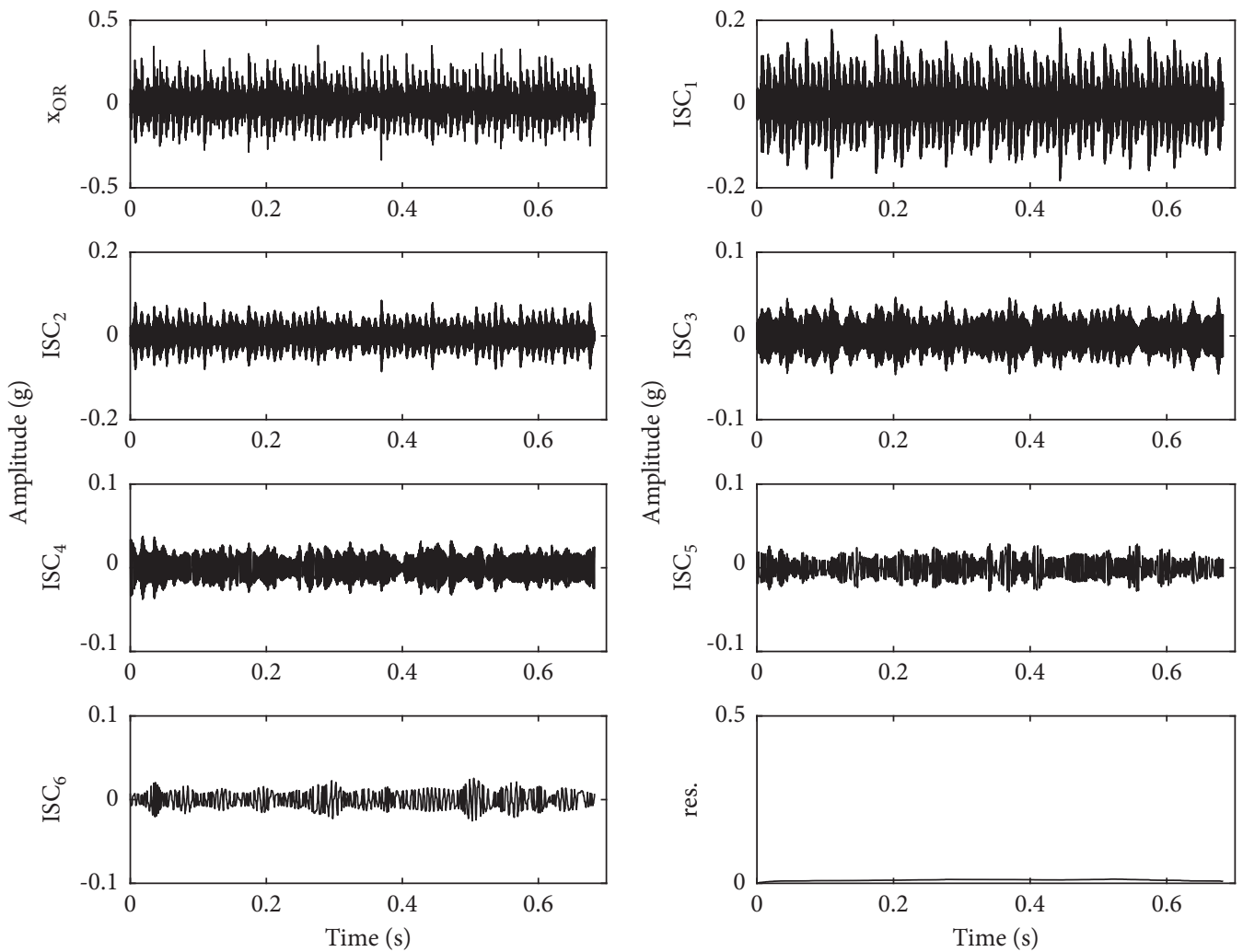

FIGURE 11: LCD decomposition results of $\mathrm{x}$-channel under outer race fault condition.
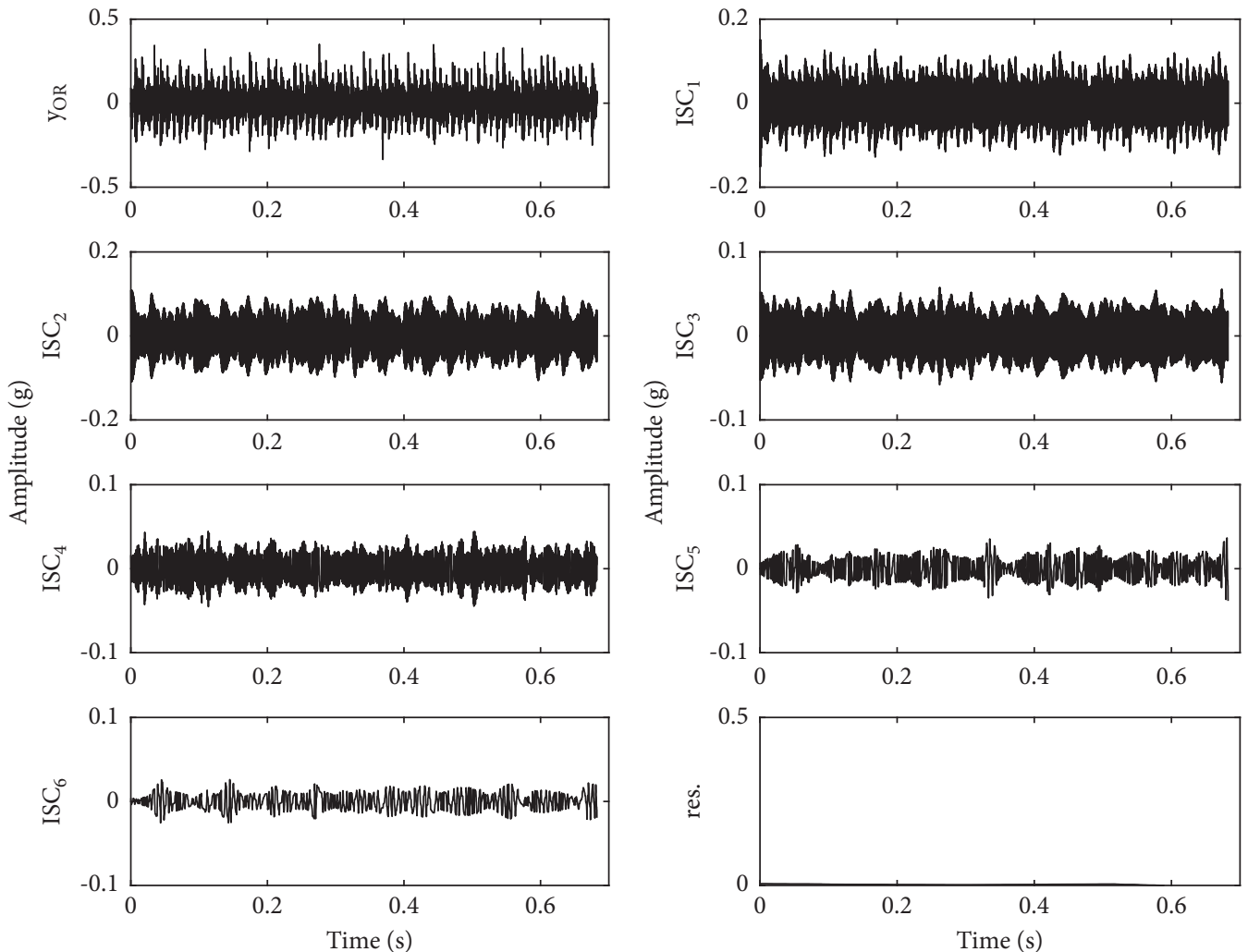

FIGURE 12: LCD decomposition results of y-channel under outer race fault condition. 
TABLE 4: Correlation coefficient of ISCs of each order under outer race fault condition.

\begin{tabular}{lccccccc}
\hline ISC order & Direction & 1 & 2 & 3 & 4 & 5 \\
\hline Correlation & $\mathrm{x}$ & $\mathbf{0 . 8 3 7 5}$ & 0.5643 & 0.2833 & 0.2490 & 0.1737 & 0.1280 \\
coefficient & $\mathrm{y}$ & $\mathbf{0 . 7 5 2 9}$ & 0.6911 & 0.3957 & 0.2071 & 0.1321 \\
ISC order & Direction & 7 & 8 & 9 & 10 & 0.0858 \\
Correlation & $\mathrm{x}$ & 0.0712 & 0.0243 & 0.0089 & 0.0001 & 11 & 12 \\
coefficient & $\mathrm{y}$ & 0.0426 & -0.001 & 0.0054 & 0.0017 & 0.0004 & 0.0008 \\
\hline
\end{tabular}

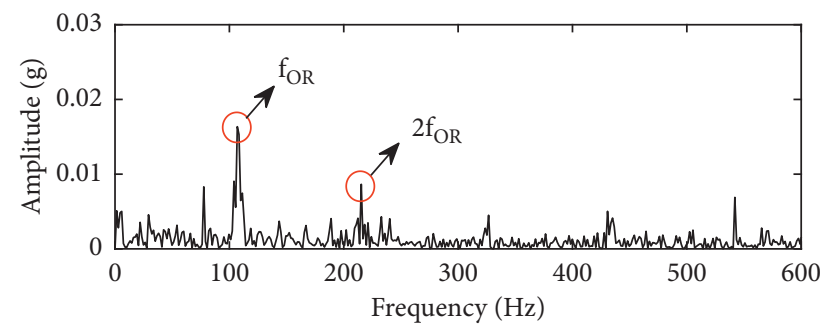

FIGURE 13: Vector LCD spectra of vector signal under outer race fault condition.
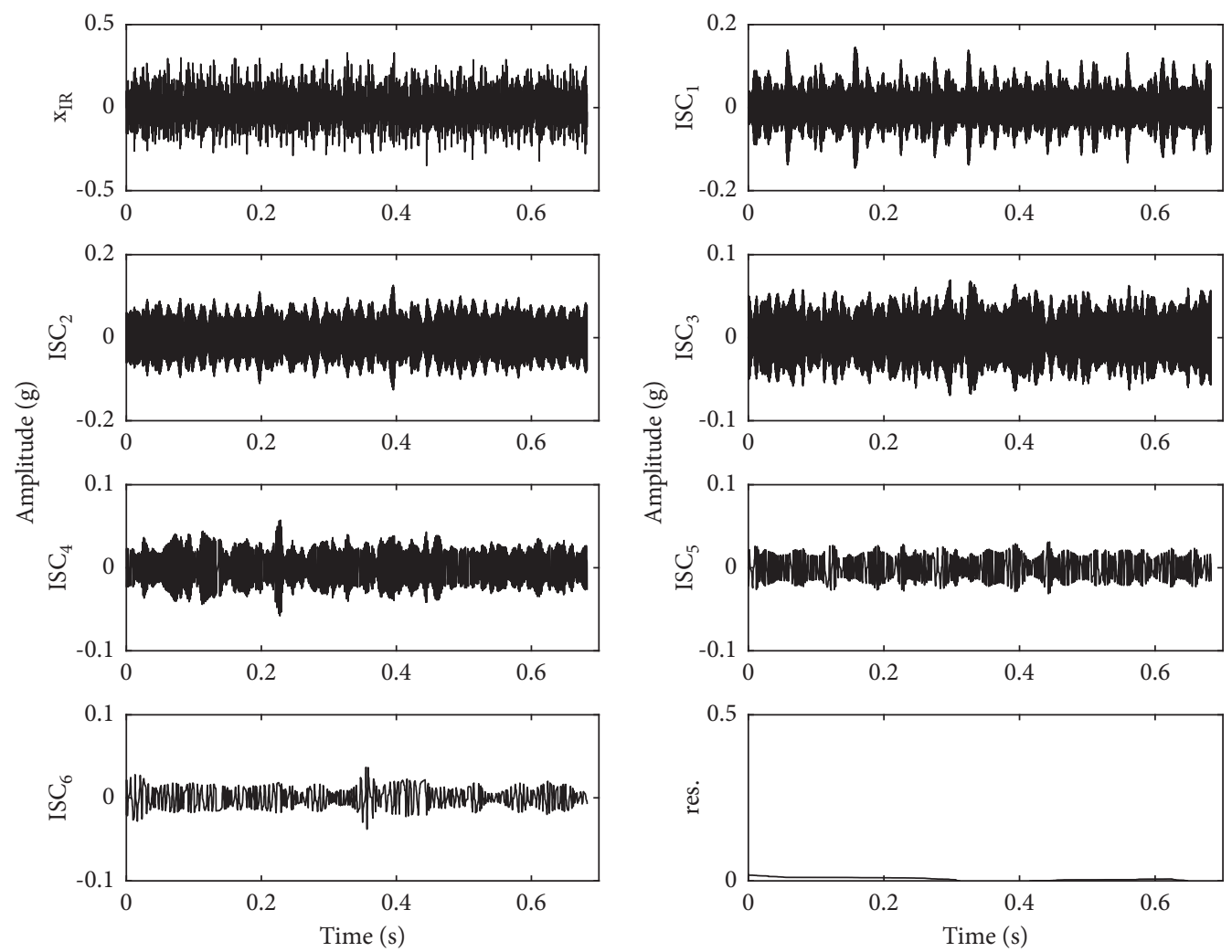

FIgURE 14: LCD decomposition results of $\mathrm{x}$-channel under inner race fault condition.

TABLE 5: Correlation coefficient of ISCs of each order under inner race fault condition.

\begin{tabular}{lcccccc}
\hline ISC order & 1 & 2 & 3 & 4 & 5 \\
\hline Correlation coefficient & $\mathbf{0 . 7 5 6 3}$ & 0.7399 & 0.4803 & 0.3197 & 0.1993 \\
ISC order & 7 & 8 & 9 & 10 & 0.1183 \\
Correlation coefficient & 0.0300 & 0.0230 & 0.0005 & 0.0005 & 11 & 0.0013 \\
\hline
\end{tabular}

The bold value is the maximum value of all ISCs (between the order 1 to 12). 


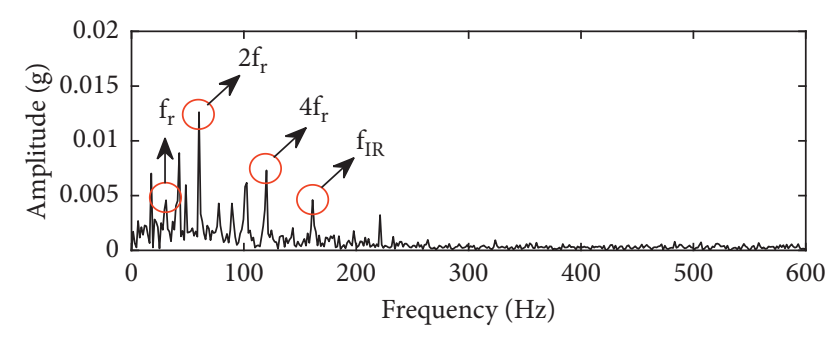

Figure 15: Vector LCD spectra of $\mathrm{x}$-signal under inner race fault condition.

\section{Conclusion}

A multiple signal processing method of rolling bearing fault diagnosis was described in this paper. By combining the full vector spectrum with local characteristic-scale decomposition, the vector LCD can fully consider homologous signals and ISCs. This method is used to synchronously handle multiple signals. The cross-correlation coefficient was introduced to choose ISCs, which simplifies the analysis of ISCs. The vector ISC displays a more complete and precise fault frequency than a single ISC. The simulation analysis and rolling bearing experimental fault diagnosis verified the effectiveness of vector LCD. With the good compatibility of vector LCD, various types of faults and machines could be diagnosed in our future work. Moreover, the signals from different types of sensors will be combined to improve the accuracy of fault diagnosis.

\section{Data Availability}

The experimental data were taken from the Case Western Reserve University bearing data center.

\section{Conflicts of Interest}

The authors declare that there are no conflicts of interest regarding the publication of this paper.

\section{Acknowledgments}

The authors would like to thank the National Key Research and Development Program of China (2016YFF0203100) and the Henan Provincial Key Science and Technology Research Project of China (202102210075). The authors also thank the Case Western Reserve University bearing data center for the bearing fault test data.

\section{References}

[1] R. Liu, B. Yang, E. Zio, and X. Chen, "Artificial intelligence for fault diagnosis of rotating machinery: a review," Mechanical Systems and Signal Processing, vol. 108, pp. 33-47, 2018.

[2] B. Zhao, X. Zhang, H. Li, and Z. Yang, "Intelligent fault diagnosis of rolling bearings based on normalized CNN considering data imbalance and variable working conditions," Knowledge-Based Systems, vol. 199, Article ID 105971, 2020.

[3] Y. Gao and D. Yu, "Intelligent fault diagnosis for rolling bearings based on graph shift regularization with directed graphs," Advanced Engineering Informatics, vol. 47, Article ID 101253, 2021.

[4] J. Cheng, Y. Yang, X. Li, and J. Cheng, "Adaptive periodic mode decomposition and its application in rolling bearing fault diagnosis," Mechanical Systems and Signal Processing, vol. 161, Article ID 107943, 2021.

[5] W. Zhu, G. Ni, Y. Cao, and H. Wang, "Research on a rolling bearing health monitoring algorithm oriented to industrial big data," Measurement, vol. 185, Article ID 110044, 2021.

[6] A. Glowacz, "Ventilation diagnosis of angle grinder using thermal imaging," Sensors, vol. 21, no. 8, p. 2853, 2021.

[7] P. Luo, N. Hu, L. Zhang, J. Shen, and Z. Cheng, "Improved phase space warping method for degradation tracking of rotating machinery under variable working conditions," Mechanical Systems and Signal Processing, vol. 157, Article ID 107696, 2021.

[8] X. Li, Y. Yang, H. Shao, X. Zhong, J. Cheng, and J. Cheng, "Symplectic weighted sparse support matrix machine for gear fault diagnosis," Measurement, vol. 168, Article ID 108392, 2021.

[9] J. Yang, D. Huang, D. Zhou, and H. Liu, "Optimal IMF selection and unknown fault feature extraction for rolling bearings with different defect modes," Measurement, vol. 157, Article ID 107660, 2020.

[10] A. Klausen, H. V. Khang, and K. G. Robbersmyr, "Multi-band identification for enhancing bearing fault detection in variable speed conditions," Mechanical Systems and Signal Processing, vol. 139, Article ID 106422, 2020.

[11] Y. Ma, J. Cheng, P. Wang, J. Wang, and Y. Yang, "Rotating machinery fault diagnosis based on multivariate multiscale fuzzy distribution entropy and Fisher score," Measurement, vol. 179, Article ID 109495, 2021.

[12] A. Glowacz, R. Tadeusiewicz, S. Legutko et al., "Fault diagnosis of angle grinders and electric impact drills using acoustic signals," Applied Acoustics, vol. 179, Article ID 108070, 2021.

[13] M. Hosseinpour-Zarnaq, M. Omid, and E. Biabani-Aghdam, "Fault Diagnosis of Tractor Auxiliary Gearbox Using Vibration Analysis and Random forest Classifier," Information Processing in Agriculture, 2021, In press.

[14] H. Pan, H. Xu, J. Zheng, J. Su, and J. Tong, "Multi-class fuzzy support matrix machine for classification in roller bearing fault diagnosis," Advanced Engineering Informatics, vol. 51, Article ID 101445, 2022.

[15] P. Tiwari and S. H. Upadhyay, "Novel self-adaptive vibration signal analysis: concealed component decomposition and its application in bearing fault diagnosis," Journal of Sound and Vibration, vol. 502, Article ID 116079, 2021.

[16] W. Ying, J. Zheng, H. Pan, and Q. Liu, "Permutation entropybased improved uniform phase empirical mode decomposition for mechanical fault diagnosis," Digital Signal Processing, vol. 117, Article ID 103167, 2021.

[17] A. Patel and P. Shakya, "Spur gear crack modelling and analysis under variable speed conditions using variational mode decomposition," Mechanism and Machine Theory, vol. 164, Article ID 104357, 2021.

[18] X. Li, J. Ma, X. Wang, J. Wu, and Z. Li, “An improved local mean decomposition method based on improved composite interpolation envelope and its application in bearing fault feature extraction," ISA Transactions, vol. 97, pp. 365-383, 2020.

[19] J. Zheng, J. Cheng, and Y. Yang, "A rolling bearing fault diagnosis approach based on LCD and fuzzy entropy," Mechanism and Machine Theory, vol. 70, pp. 441-453, 2013. 
[20] H. Liu, X. Wang, and C. Lu, "Rolling bearing fault diagnosis based on LCD-TEO and multifractal detrended fluctuation analysis," Mechanical Systems and Signal Processing, vol. 6061, pp. 273-288, 2015.

[21] L. Wang and Z. Liu, "An improved local characteristic-scale decomposition to restrict end effects, mode mixing and its application to extract incipient bearing fault signal," $\mathrm{Me}$ chanical Systems and Signal Processing, vol. 156, Article ID 107657, 2021.

[22] C. Yang, J. Ma, X. Wang, X. Li, Z. Li, and T. Luo, "A Novel Based-Performance Degradation Indicator RUL Prediction Model and its Application in Rolling Bearing," ISA Transactions, 2021.

[23] P. Zhang, T. Li, G. Wang et al., "Multi-source information fusion based on rough set theory: a review," Information Fusion, vol. 68, pp. 85-117, 2021.

[24] S. Liu, "A modified low-speed balancing method for flexible rotors based on holospectrum," Mechanical Systems and Signal Processing, vol. 21, no. 1, pp. 348-364, 2007.

[25] S. Liu and L. Qu, "A new field balancing method of rotor systems based on holospectrum and genetic algorithm," Applied Soft Computing, vol. 8, no. 1, pp. 446-455, 2008.

[26] L. Qu, Y. Liao, J. Lin, and M. Zhao, "Investigation on the subsynchronous pseudo-vibration of rotating machinery," Journal of Sound and Vibration, vol. 423, pp. 340-354, 2018.

[27] L. Qu, X. Liu, G. Peyronne, and Y. Chen, "The holospectrum: a new method for rotor surveillance and diagnosis," Mechanical Systems and Signal Processing, vol. 3, no. 3, pp. 255-267, 1989.

[28] A. Muszynska and P. Goldman, "Application of full spectrum to rotating machinery diagnostics," Orbit, vol. 1, no. 20, pp. 17-21, 1999.

[29] T. H. Patel and A. K. Darpe, "Application of full spectrum analysis for rotor fault diagnosis,"Springer, New York, NY, USA.

[30] T. Patel and A. Darpe, "Use of full spectrum cascade for rotor rub identification," Advances in Vibration Engineering, vol. 8, pp. 139-151, 2009.

[31] X. Zhao, T. H. Patel, and M. J. Zuo, "Multivariate EMD and full spectrum based condition monitoring for rotating machinery," Mechanical Systems and Signal Processing, vol. 27, pp. 712-728, 2012.

[32] J. Han and L. Shi, Full Vector Spectrum Technology and its Enginnering Application, China Machine Press, Beijing,China, 2008.

[33] L. Chen, J. Han, W. Lei, Z. Guan, and Y. Gao, "Prediction model of vibration feature for equipment maintenance based on full vector spectrum," Shock and Vibration, vol. 2017, Article ID 6103947, 8 pages, 2017.

[34] X. Gong, L. Ding, and W. Du, "Application of the full vector spectrum to local rub-impact fault diagnosis in rotor systems," Journal of Residuals Science \& Technology, vol. 13, no. 8, 2016.

[35] H. Li, X. M. Dong, W. S. Hao, A. G. Liu, X. D. Yin, and A. J. Wang, "Applying full vector spectrum for electric hoist gearbox fault diagnosis," Applied Mechanics and Materials, vol. 365-366, pp. 725-728, 2013.

[36] L. Chen, J. Han, W. Lei, Y. Cui, and Z. Guan, "Full-vector signal acquisition and information fusion for the fault prediction," International Journal of Rotating Machinery, vol. 2016, Article ID 5980802, 7 pages, 2016.

[37] X. Gong, L. Ding, W. Du, and H. Wang, "Gear fault diagnosis using dual channel data fusion and EEMD method," Procedia Engineering, vol. 174, pp. 918-926, 2017.
[38] H. Yu, H. Li, Y. Li, and Y. Li, "A novel improved full vector spectrum algorithm and its application in multi-sensor data fusion for hydraulic pumps," Measurement, vol. 133, pp. 145-161, 2019.

[39] J. Cheng, J. Zheng, and Y. Yang, “A new non-stationary signal analysis approach-the local characteristic-scale decomposition method," Journal of Vibration Engineering, vol. 25, no. 2, pp. 215-220, 2012.

[40] J. Han and L. Shi, "Study on space precession and vibration characteristic of high-speed rotary shaft," Journal of Vibration Engineering, vol. 17, no. 3, pp. 69-72, 2004.

[41] W. A. Smith and R. B. Randall, "Rolling element bearing diagnostics using the Case Western Reserve University data: a benchmark study," Mechanical Systems and Signal Processing, vol. 64-65, pp. 100-131, 2015. 